\title{
STABILITY OF NONAXISYMMETRIC FERROFLUID FLOW IN ROTATING CYLINDERS WITH MAGNETIC FIELD
}

\author{
JITENDER SINGH AND RENU BAJAJ
}

Received 8 June 2005 and in revised form 17 October 2005

Effect of an axially applied magnetic field on the stability of a ferrofluid flow in an annular space between two coaxially rotating cylinders with nonaxisymmetric disturbances has been investigated numerically. The critical value of the ratio $\Omega^{*}$ of angular speeds of the two cylinders, at the onset of the first nonaxisymmetric mode of disturbance, has been observed to be affected by the applied magnetic field.

\section{Introduction}

The problem of a viscous flow in between two coaxially rotating cylinders has been of wide interest since Taylor's classical work [15]. He found experimentally that the first instability occurred via a stationary, axisymmetric disturbance of the Couette flow in the form of cellular toroidal vortices, spaced regularly along the axis of the cylinders. His theoretical findings were in excellent agreement with his experimental observations. Since then, this problem has been greatly studied, both experimentally and theoretically. A rich variety of patterns occur when the relative velocity of inner cylinder is increased. The attractive feature of this experiment to exhibit a large number of different flows has made this problem important.

This kind of flow has been used in many practical applications such as in viscosity measurements and in modelling of jet streams. The linear stability of the Couette flow with axisymmetric disturbance has been studied extensively by Chandrasekhar [3], Harris and Reid [9], Di Prima [6], Donnelly [8], Coles [5], and many others.

Recently, the problem of stability of magnetic fluid in Taylor-Couette system has gained popularity. The Couette flow in ferrofluids has given rise to numerous applications in making high-quality ferrofluid seals for rotary shafts in engineering and in producing high-speed silent printers [12]. The magnetic properties of ferrofluids [2,12] and features of the Couette flow have made the problem interesting for further investigation. The stability of a ferrofluid flow in Taylor-Couette system in the presence of magnetic field has been studied by Niklas et al. [11]. They have studied the problem linearly and have found the explicit finite-difference numerical solution of the full nonlinear time-dependent field equations in the presence of general magnetic field with small-gap approximations. 
Singh and Bajaj [14] have discussed the stability of Couette flow of ferrofluid in the presence of an axially applied magnetic field. They have taken into account the axisymmetric perturbations of magnetic field in the ferrofluid in the gap between two uniformly rotating cylinders. They have found that magnetic field perturbations cause the delay of the onset of instability in the Couette flow. When the cylinders are counter-rotating, the onset of instability can occur via a nonaxisymmetric mode.

The stability of Couette flow with nonaxisymmetric disturbance was first considered by Di Prima [7]. Using Galerkin method, he solved the problem linearly with narrowgap approximations and for nonnegative values of the ratio $\Omega^{*}$ of angular speed of outer cylinder to that of inner cylinder. He found that the critical speed increases on increasing the azimuthal wave number. Krueger et al. [10] carried out the complete linear stability analysis of the Couette flow with nonaxisymmetric disturbance for narrow- as well as for wide-gap considerations between the cylinders. They found that there exists a critical value of the parameter $\Omega^{*}$, of approximately -0.78 , above which the critical disturbance is axisymmetric and below which it is nonaxisymmetric.

Chang et al. [4] have studied the stability of ferrofluid flow between two concentric rotating cylinders in the presence of constant axial magnetic field. They have discussed the linear stability of this problem in general. They have found that the onset of instability could be dominated by nonaxisymmetric mode only when the value of parameter $\Omega^{*}$ is sufficiently negative even if the strength of the magnetic field is very large.

In the present analysis, we have taken into consideration the nonaxisymmetric disturbances in the Couette flow of a viscous, incompressible, Newtonian ferrofluid in the presence of an axially applied magnetic field. The magnetic field perturbations in ferrofluid in the gap between two uniformly rotating cylinders have been considered and their effect on the stability of flow for the onset of the first nonaxisymmetric mode of disturbance has been investigated.

\section{Formulation}

We consider a viscous, incompressible, Newtonian ferrofluid flow in an annular region between two coaxial cylinders of radii $r_{1}$ and $r_{2},\left(r_{1}<r_{2}\right)$, respectively, rotating with uniform angular speeds $\Omega_{1}$ and $\Omega_{2}$, respectively, about their common axis taken as $z$-axis. The cylinders are assumed to be of infinite length. We have used cylindrical polar coordinates $(r, \theta, z)$, which denote, respectively, radial, azimuthal, and axial coordinates. A constant magnetic field $\mathbf{h}_{0}=\left(0,0, h_{0}\right)$ is applied to this system along the axis of the cylinders. Then the governing equations [13] for the flow are

$$
\begin{gathered}
\frac{\partial \mathbf{u}}{\partial t}+\mathbf{u} \cdot \nabla \mathbf{u}=-\frac{1}{\rho} \nabla p+\nu \nabla^{2} \mathbf{u}+\frac{\mu_{0}}{\rho} \mathbf{m} \cdot \nabla \mathbf{h}+\frac{\mu_{0}}{2 \rho} \nabla \times(\mathbf{m} \times \mathbf{h}), \\
\frac{\partial \mathbf{m}}{\partial t}+\mathbf{u} \cdot \nabla \mathbf{m}=\frac{1}{2}(\nabla \times \mathbf{u}) \times \mathbf{m}-\alpha\left(\mathbf{m}-\mathbf{m}_{0}\right)-\beta \mathbf{m} \times(\mathbf{m} \times \mathbf{h}), \\
\nabla \cdot \mathbf{u}=0, \quad \nabla \times \mathbf{h}=0, \quad \nabla \cdot(\mathbf{m}+\mathbf{h})=0,
\end{gathered}
$$

where $\mathbf{u}, \mathbf{h}$, and $\mathbf{m}$ are velocity, magnetic field, and magnetization, respectively, for the ferrofluid. $p$ is the total pressure of the ferrofluid, $\rho$ is its density, $v$ is the kinematic 
viscosity of ferrofluid, and $\mu_{0}$ is the magnetic permeability of free space. $\alpha=k_{b} T_{b} / 3 V_{h} \eta$, $\beta=\mu_{0} / 6 \varphi \eta, \eta=\rho v$, where $k_{b}, T_{b}, \varphi$, and $V_{h}$ are Boltzmann constant, temperature of the ferrofluid, volume fraction of the ferromagnetic particles, and hydrodynamic volume of a ferrocolloid particle, respectively. $\mathbf{m}_{0}$ is the equilibrium magnetization of the ferrofluid, which is related to the equilibrium magnetic field $\mathbf{h}_{0}$ by the Langevin formula

$$
\mathbf{m}_{0}=n \bar{m}(\operatorname{coth} \psi-1 / \psi) \frac{\mathbf{h}_{0}}{\left|\mathbf{h}_{0}\right|}
$$

where $n$ is the number density of ferromagnetic particles and $\bar{m}$ is the magnetic moment of single ferromagnetic particle, $\psi=\mu_{0}\left(\bar{m} h_{0} / k_{b} T_{b}\right)$ is the magnetic field parameter.

In system (2.1), velocity field $\mathbf{u}$ satisfies the following boundary conditions:

$$
\mathbf{u}=\left(0, r \Omega_{j}, 0\right) \quad \text { at } r=r_{j}, \text { for } j=1,2
$$

Magnetic induction field $\mathbf{b}\left(=\mu_{0}(\mathbf{m}+\mathbf{h})\right)$ and magnetic field $\mathbf{h}$ satisfy

$$
\widehat{\mathbf{n}} \cdot[\mathbf{m}+\mathbf{h}]=0, \quad \hat{\mathbf{n}} \times[\mathbf{h}]=0, \quad \text { at } r=r_{1}, r_{2},
$$

where $\hat{\mathbf{n}}$ denotes outward drawn unit normal to the curved surface of outer cylinder and $[\mathbf{m}+\mathbf{h}]$ and $[\mathbf{h}]$ denote the difference in $\mathbf{m}+\mathbf{h}$ and $\mathbf{h}$, respectively, across the boundaries.

System (2.1) along with boundary conditions (2.3)-(2.4) has a circular Couette flow as a stationary solution:

$$
\mathbf{u}=(0, r \Omega, 0), \quad \mathbf{h}=\left(0,0, h_{0}\right), \quad \mathbf{m}=\left(0,0, m_{0}\right), \quad p=p_{0},
$$

where $\Omega=A+B / r^{2}, A=\Omega_{1}\left(\Omega^{*}-\xi^{2}\right) /\left(1-\xi^{2}\right), B=r_{1}{ }^{2} \Omega_{1}\left(1-\Omega^{*}\right) /\left(1-\xi^{2}\right), \Omega^{*}=$ $\Omega_{2} / \Omega_{1}, \xi=r_{1} / r_{2}$, and $p_{0}=\rho \int r \Omega^{2} d r$.

2.1. Perturbation equations. Due to the motion of the ferrofluid particles in the TaylorCouette flow, the magnetic field $\mathbf{h}$ inside the ferrofluid changes. In stationary state, ferrofluid is magnetized in direction of applied magnetic field but due to its motion, magnetization vector $\mathbf{m}$ in the nonequilibrium state is no longer parallel to the direction of magnetic field. To represent the nonequilibrium state, we superimpose a three-dimensional disturbance on the stationary solution (2.5) in the form

$$
\begin{gathered}
\mathbf{u}=\left(u_{r}, r \Omega+u_{\theta}, u_{z}\right), \quad \mathbf{h}=\left(h_{r}, h_{\theta}, h_{0}+h_{z}\right), \\
\mathbf{m}=\left(m_{r}, m_{\theta}, m_{0}+m_{z}\right), \quad p=p_{0}+p_{1} .
\end{gathered}
$$

Let $R=r_{2}-r_{1}$ be the gap width of fluid region in between the cylinders. Introducing $R, R^{2} / \nu, \nu / R, \rho v^{2} / R^{2}, \nu\left(2 \rho / \mu_{0}\right)^{1 / 2} / R$, as the characteristic scales for length, time, speed, pressure, and magnetic field, respectively, we nondimensionalize the system (2.1). The solution for the linearized, dimensionless system can be assumed in the following normal 
modes:

$$
\begin{aligned}
\mathbf{u} & =\left(0, \frac{r \Omega R^{2}}{\nu}, 0\right)+\left(\hat{u}_{r}(r), \hat{u}_{\theta}(r), \hat{u}_{z}(r)\right) e^{i(\omega t+\sigma \theta+k z)}, \\
\mathbf{h} & =\left(0,0, \mathcal{H} h_{0}\right)+\left(\hat{h}_{r}(r), \hat{h}_{\theta}(r), \hat{h}_{z}(r)\right) e^{i(\omega t+\sigma \theta+k z)}, \\
\mathbf{m} & =\left(0,0, \mathcal{H} m_{0}\right)+\left(\hat{m}_{r}(r), \hat{m}_{\theta}(r), \hat{m}_{z}(r)\right) e^{i(\omega t+\sigma \theta+k z)}, \\
p & =\frac{R^{2} p_{0}}{\left(\rho v^{2}\right)}+\hat{p}(r) e^{i(\omega t+\sigma \theta+k z)},
\end{aligned}
$$

where $\mathcal{H}=R\left(\mu_{0} / 2 \rho\right)^{1 / 2} / \nu, k$ is assumed to be real, and $\sigma$ a nonnegative integer. The parameter $\omega$ is complex in general.

Substituting (2.7) in the linearized system, we obtain the following equations:

$$
\begin{aligned}
& \left(D D^{*}-k^{2}\right) \hat{u}_{r}=D \hat{p}+\left(i \omega+\frac{i \sigma R^{2}}{\nu} \Omega+\frac{\sigma^{2}}{r^{2}}\right) \hat{u}_{r}+2\left(\frac{i \sigma}{r^{2}}-\frac{R^{2}}{\nu} \Omega\right) \hat{u}_{\theta}-i k \mathcal{H} h_{0} \hat{m}_{r}, \\
& \left(D D^{*}-k^{2}\right) \hat{\mathcal{u}}_{\theta}=\frac{i \sigma}{r} \hat{p}+\left(i \omega+\frac{i \sigma R^{2}}{v} \Omega \frac{\sigma^{2}}{r^{2}}\right) \hat{\mathcal{u}}_{\theta}+2\left(-\frac{i \sigma}{r^{2}}-\frac{T \nu}{4 \Omega_{1} R^{2}}\right) \hat{u}_{r}-i k \mathscr{H} h_{0} \hat{m}_{\theta}, \\
& \left(D D^{*}-k^{2}\right) \hat{u}_{z}=i k \hat{p}+\left(i \omega+\frac{i \sigma R^{2}}{v} \Omega+\frac{\sigma^{2}}{r^{2}}\right) \hat{u}_{z}-i k \mathcal{H} h_{0} \hat{m}_{z} \\
& -\mathscr{H}\left(m_{0}+h_{0}\right)\left(D^{*} \hat{h}_{r}+\frac{i \sigma}{r} \hat{h}_{\theta}+i k \hat{h}_{z}\right) \\
& {\left[i \omega+\frac{i \sigma R^{2} \Omega}{v}+\frac{R^{2} \alpha}{v}+\frac{R^{2} \beta m_{0} h_{0}}{v}\right] \hat{m}_{r}+\frac{R^{2} r D \Omega}{2 v} \hat{m}_{\theta}} \\
& =\frac{\mathscr{H} m_{0}}{2}\left(i k \hat{u}_{r}-D \hat{u}_{z}\right)+\frac{\beta R^{2} m_{0}^{2}}{\nu} \hat{h}_{r} \\
& {\left[i \omega+\frac{i \sigma R^{2} \Omega}{v}+\frac{R^{2} \alpha}{v}+\frac{R^{2} \beta m_{0} h_{0}}{v}\right] \hat{m}_{\theta}-\frac{R^{2} r D \Omega}{2 v} \hat{m}_{r}} \\
& =\frac{i \mathscr{H} m_{0}}{2}\left(k \hat{u}_{\theta}-\frac{\sigma}{r} \hat{u}_{z}\right)+\frac{\beta R^{2} m_{0}^{2}}{\nu} \hat{h}_{\theta} \\
& \hat{m}_{z}=0, \quad D^{*} \hat{u}_{r}=-\frac{i \sigma}{r} \hat{u}_{\theta}-i k \hat{u}_{z}, \quad \hat{h}_{\theta}=\frac{\sigma}{k r} \hat{h}_{z}, \quad D \hat{h}_{z}=i k \hat{h}_{r}, \\
& D^{*}\left(\hat{m}_{r}+\hat{h}_{r}\right)+\frac{i \sigma}{r}\left(\hat{m}_{\theta}+\hat{h}_{\theta}\right)+i k\left(\hat{m}_{z}+\hat{h}_{z}\right)=0,
\end{aligned}
$$

where $D \equiv d / d r$ and $D^{*} \equiv d / d r+1 / r$. The dimensionless parameter $T=-4 A \Omega_{1} R^{4} / \nu^{2}$ is the standard Taylor number.

System (2.8) satisfies the boundary conditions

$$
\hat{u}_{r}=\hat{u}_{\theta}=\hat{u}_{z}=\hat{m}_{r}+\hat{h}_{r}=\hat{h}_{z}=0, \quad \text { at } r=r_{1}^{*}, r_{2}^{*} \text {, }
$$

where $r_{1}^{*}=r_{1} / R$ and $r_{2}^{*}=r_{2} / R$. 
2.2. Solution. The system (2.8) has been reduced to a system of first-order ordinary differential equations by using the transformations

$$
\begin{gathered}
\hat{u}_{r}=X_{1}, \quad D^{*} \hat{u}_{r}=X_{6}+\hat{p}, \quad \hat{u}_{\theta}=X_{2}, \quad D^{*} \hat{u}_{\theta}=X_{7}, \\
\hat{u}_{z}=X_{3}, \quad D \hat{u}_{z}=X_{8}-\frac{\mathcal{H}\left(m_{0}+h_{0}\right)}{i k} X_{10}, \\
\hat{m}_{r}+\hat{h}_{r}=X_{4}, \quad D^{*}\left(\hat{m}_{r}+\hat{h}_{r}\right)=X_{9}, \quad \hat{h}_{z}=X_{5}, \quad D \hat{h}_{z}=X_{10} .
\end{gathered}
$$

We obtain the following system of ten first-order ODEs:

$$
\begin{aligned}
& D^{*} X_{1}=-\frac{i \sigma}{r} X_{2}-i k X_{3}, \quad D^{*} X_{2}=X_{7}, \quad D X_{3}=X_{8}-\frac{\mathscr{H}\left(m_{0}+h_{0}\right)}{i k} X_{10}, \\
& D^{*} X_{4}=X_{9}, \quad D X_{5}=X_{10}, \\
& D X_{6}=\left[i \omega+\frac{i \sigma R^{2}}{\nu} \Omega+\frac{\sigma^{2}}{r^{2}}+k^{2}\left(1+\frac{\mathscr{H}^{2} m_{0} h_{0} g_{1}}{2}\right)\right] X_{1} \\
& +\left[2\left(\frac{i \sigma}{r^{2}}-\frac{R^{2} \Omega}{v}\right)+\frac{k^{2} \mathscr{H}^{2} m_{0} h_{0} g_{2}}{2}\right] X_{2}-\frac{\sigma \mathcal{H}^{2} m_{0} h_{0} g_{2}}{2 r} X_{3}-\frac{i \sigma \mathscr{H} R^{2} \beta m_{0}^{2} h_{0} g_{2}}{\nu r} X_{5} \\
& +\frac{i k m_{0} h_{0} \mathscr{H}^{2} g_{1}}{2} X_{8}-\mathscr{H} h_{0} g_{1}\left[\frac{R^{2} \beta m_{0}^{2}}{\nu}+\frac{\mathscr{H}^{2} m_{0}\left(m_{0}+h_{0}\right)}{2}\right] X_{10} \text {, } \\
& D X_{7}=\left[-\frac{T \nu}{2 \Omega_{1} R^{2}}-\frac{2 i \sigma}{r^{2}}-\frac{k^{2} \mathscr{H}^{2} m_{0} h_{0} g_{2}}{2}\right] X_{1} \\
& +\left[i \omega+\frac{i \sigma R^{2}}{\nu} \Omega+\frac{2 \sigma^{2}}{r^{2}}+k^{2}\left(1+\frac{\mathscr{H}^{2} m_{0} h_{0} g_{1}}{2}\right)\right] X_{2}+\frac{\sigma k}{r}\left[1-\frac{\mathscr{H}^{2} m_{0} h_{0} g_{1}}{2}\right] X_{3} \\
& -\frac{i \sigma \mathscr{H} R^{2} \beta m_{0}^{2} h_{0} g_{1}}{\nu r} X_{5}-\frac{i \sigma}{r} X_{6}-\frac{i k \mathscr{H}^{2} m_{0} h_{0} g_{2}}{2} X_{8} \\
& +\operatorname{Hh}_{0} g_{2}\left[\frac{R^{2} \beta m_{0}^{2}}{v}+\frac{\mathscr{H}^{2} m_{0}\left(m_{0}+h_{0}\right)}{2}\right] X_{10} \\
& D^{*} X_{8}=\frac{\sigma k}{r} X_{2}+\left[i \omega+\frac{i \sigma R^{2}}{v} \Omega+\frac{\sigma^{2}}{r^{2}}+2 k^{2}\right] X_{3} \\
& -i \mathscr{H}\left(m_{0}+h_{0}\right)\left(\frac{\sigma^{2}+k^{2} r^{2}}{k r^{2}}\right) X_{5}-i k X_{6} \text {, } \\
& D X_{9}=i k\left(D f_{3}-\frac{f_{3}}{r}\right) X_{1}-i k\left(D f_{4}-\frac{f_{4}}{r}\right) X_{2} \\
& +\left[\frac{i \sigma^{2}}{k} D\left(\frac{f_{4}}{r^{2}}\right)-\left(i \omega+\frac{i \sigma R^{2}}{v} \Omega+\frac{\sigma^{2}}{r^{2}}+k^{2}\right) f_{3}\right] X_{3}+\left(D f_{2}-\frac{f_{2}}{r}\right) X_{4} \\
& +\left[i \mathscr{H}\left(m_{0}+h_{0}\right) \frac{\left(\sigma^{2}+k^{2} r^{2}\right)}{k r^{2}}-D f_{1}\right] X_{5}+i k f_{3} X_{6}-i k f_{4} X_{7} \\
& +\left[\frac{i \sigma}{r} f_{4}-D f_{3}+\frac{f_{3}}{r}\right] X_{8}+f_{2} X_{9}-\left[f_{1}+\frac{\sigma \mathcal{H}\left(m_{0}+h_{0}\right)}{k r} f_{4}\right] X_{10},
\end{aligned}
$$




$$
\begin{aligned}
D^{*} X_{10}= & \frac{k^{2} \mathscr{H} m_{0} D g_{1}}{2 \Delta} X_{1}+\frac{k^{2} \mathscr{H} m_{0} D g_{2}}{2 \Delta} X_{2} \\
& +\frac{i k \mathscr{H} m_{0}}{2 \Delta}\left[\frac{i \sigma}{r} D g_{2}+\left(i \omega+\frac{i \sigma R^{2}}{v} \Omega+\frac{\sigma^{2}}{r^{2}}+k^{2}\right) g_{1}\right] X_{3} \\
& +\frac{1}{\Delta}\left[\frac{\mathscr{H}^{2} m_{0}\left(m_{0}+h_{0}\right)}{2} \frac{\left(\sigma^{2}+k^{2} r^{2}\right)}{r^{2}}-\frac{i \sigma R^{2} \beta m_{0}^{2}}{v} D^{*}\left(\frac{g_{2}}{r}\right)\right] X_{5} \\
& +\frac{k^{2} \mathscr{H} m_{0} g_{1}}{2 \Delta} X_{6}+\frac{k^{2} \mathscr{H} m_{0} g_{2}}{2 \Delta} X_{7}+\frac{i k \mathscr{H} m_{0}}{2 \Delta}\left(D g_{1}+\frac{i \sigma}{r} g_{2}\right) X_{8}+\frac{i k}{\Delta} X_{9} \\
& -\frac{1}{\Delta}\left[\frac{R^{2} \beta m_{0}^{2}}{v}+\frac{\mathscr{H}^{2} m_{0}\left(m_{0}+h_{0}\right)}{2}\right]\left(D g_{1}+\frac{i \sigma}{r} g_{2}\right) X_{10},
\end{aligned}
$$

where

$$
\begin{gathered}
g_{1}=\frac{i \omega+\left(i \sigma R^{2} / \nu\right) \Omega+\left(R^{2} / \nu\right)\left(\alpha+\beta m_{0} h_{0}\right)}{\left[i \omega+\left(i \sigma R^{2} / \nu\right) \Omega+\left(R^{2} / \nu\right)\left(\alpha+\beta m_{0} h_{0}\right)\right]^{2}+R^{4} r^{2}(D \Omega)^{2} / 04 \nu^{2}}, \\
g_{2}=-\frac{R^{2} r D \Omega / 2 \nu}{\left[i \omega+\left(i \sigma R^{2} / \nu\right) \Omega+\left(R^{2} / \nu\right)\left(\alpha+\beta m_{0} h_{0}\right)\right]^{2}+R^{4} r^{2}(D \Omega)^{2} / 4 \nu^{2}}, \\
\Delta=1+\left[\frac{R^{2} \beta m_{0}^{2}}{\nu}+\frac{\mathcal{H}^{2} m_{0}\left(m_{0}+h_{0}\right)}{2}\right] g_{1}, \\
f_{1}=\frac{i\left(\sigma^{2}+k^{2} r^{2}\right)}{k r^{2}}+\frac{i \sigma^{2} R^{2} \beta m_{0}^{2}}{\nu k r^{2}}\left[\frac{\left(g_{1}^{2}+g_{2}^{2}\right) \Delta-g_{2}^{2}}{g_{1} \Delta}\right], \\
f_{2}=\frac{i \sigma g_{2}}{r g_{1}}\left(\frac{\Delta-1}{\Delta}\right), \quad f_{3}=\frac{i \sigma \mathcal{H} m_{0} g_{2}}{2 r \Delta}, \quad f_{4}=\frac{i \sigma m_{0}}{2 r g_{1} \Delta}\left[\left(g_{1}^{2}+g_{2}^{2}\right) \Delta-g_{2}^{2}\right] .
\end{gathered}
$$

The associated boundary conditions in terms of the new variables can be written as

$$
X_{1}=X_{2}=X_{3}=X_{4}=X_{5}=0 \quad \text { at } r=r_{1}^{*}, r_{2}^{*} \text {. }
$$

The system of (2.11) along with ten boundary conditions given by (2.13) leads to a two-point boundary value problem that has been solved by using a shooting method $[9,14]$.

The five linearly independent solutions $Y_{j}(1 \leq j \leq 5)$ (where each $Y_{j}$ is a column matrix with ten rows) for the system (2.11) can be constructed such that they satisfy the initial conditions

$$
\mathbf{Y}=\left(\begin{array}{ll}
\mathbf{O}_{5} & \mathbf{I}_{5}
\end{array}\right)^{t} \text { at } r=r_{1}^{*},
$$

where $\mathbf{Y}=\left(Y_{1} Y_{2} \cdots Y_{5}\right)$, superscript $t$ denotes the matrix transpose, $\mathbf{O}_{5}$ and $\mathbf{I}_{5}$ are, respectively, zero matrix and identity matrix of order five.

The functions $Y_{j}$ 's have been evaluated by solving the system (2.11) together with initial conditions (2.14), using fourth-order Runge-Kutta method. We define $\mathbf{X}$ as the 
column vector $\left(X_{1} X_{2} \cdots X_{9} X_{10}\right)^{t}$. Then any other solution of the system (2.11) satisfying the initial conditions (2.14) at $r=r_{1}^{*}$ is a linear combination of these five solutions $Y_{j}$ 's, that is,

$$
\mathbf{X}=\mathbf{Y} \cdot \mathbf{C},
$$

where $\mathbf{C}$ is an arbitrary constant column matrix with five rows. This solution should satisfy the boundary conditions required at $r=r_{2}$, that is, the functions in first five rows of the column matrix $\mathbf{X}$ should vanish at $r=r_{2}$. We decompose the matrix $\mathbf{Y}$ as $\mathbf{Y}=$ $\left(\begin{array}{lll}Z_{1} & Z_{2}\end{array}\right)^{t}$ such that each of the matrices $Z_{1}, Z_{2}$ is of order five, then (2.15) is equivalent to

$$
\mathbf{X}=\left(\begin{array}{ll}
\mathbf{Z}_{1} & \mathbf{Z}_{2}
\end{array}\right)^{t} \cdot \mathbf{C},
$$

with

$$
\mathrm{Z}_{1} \cdot \mathbf{C}=\mathbf{O} \quad \text { at } r=r_{2}^{*},
$$

where $\mathbf{O}$ is a zero matrix of order $5 \times 1$. Equation (2.17) is homogeneous in $\mathbf{C}$. For a nontrivial solution of $\mathbf{C}$, we have the determinant

$$
\left|\mathbf{Z}_{1}\right|=0 \quad \text { at } r=r_{2}^{*} .
$$

This is the required secular equation which involves unknowns: the Taylor number $T$, the axial wave number $k$, the azimuthal wave number $\sigma$, and the angular frequency of disturbance $\omega$. The marginal state is characterized by vanishing of the imaginary part of parameter $\omega$. We have solved the secular equation (2.18) numerically to calculate the critical Taylor number $T_{c}$ for fixed values of the parameters $\xi, \Omega^{*}, \varphi$, and $\psi$.

\section{Results and discussion}

The numerical results have been obtained for a ferrofluid of magnetite with carrier liquid as a diester. Its physical properties can be seen in [1]. We have fixed the ratio of the radius of the inner cylinder to that of outer cylinder, $\xi=0.95$, and the volume fraction of ferromagnetic particles, $\varphi=0.2$, for numerical calculations.

There exists a critical value $\Omega_{c}^{*}$ of the ratio $\Omega^{*}$ of angular speeds of the two cylinders, above which the onset mode of instability is axisymmetric and below which it is nonaxisymmetric. In the absence of magnetic field, the value $\Omega_{c}^{*}$ has been found to be approximately -0.75 . Krueger et al. [10] have found this value as -0.78 . With axially applied magnetic field, the critical value $\Omega_{c}^{*}$ varies with the parameter $\psi$ characterizing the magnitude of applied magnetic field. The critical values of the parameter $\Omega^{*}$ and the corresponding values of critical Taylor number $T_{c}$ obtained for various values of magnetic field parameter $\psi$ at $\sigma=0,1$ have been listed in Table 3.1. Figure 3.1 shows the plot of $\Omega_{c}^{*}$ with respect to $\psi$. It has been observed that for low and high values of $\psi, \Omega_{c}^{*}$ is close to -0.75 but for intermediate values of $\psi$, it differs from -0.75 significantly. Initially, $\Omega_{c}^{*}$ increases with increase in $\psi$, attains a maximum, and then starts decreasing with further increase in $\psi$ until its value is close to -0.75 . Chang et al. [4] have given the critical 
Table 3.1

\begin{tabular}{cccc}
\hline $\begin{array}{l}\text { Magnetic field } \\
\text { parameter } \psi\end{array}$ & $\Omega_{c}^{*}$ & $T_{c}(\sigma=0)$ & $T_{c}(\sigma=1)$ \\
\hline 0 & -0.75 & 12281 & 12272 \\
0.5 & -0.73 & 12215 & 12214 \\
1 & -0.71 & 12890 & 12885 \\
2 & -0.67 & 14295 & 14294 \\
3 & -0.66 & 15573 & 15573 \\
4 & -0.67 & 16822 & 16809 \\
6 & -0.68 & 17784 & 17772 \\
8 & -0.69 & 18220 & 18212 \\
10 & -0.70 & 18527 & 18518 \\
15 & -0.72 & 19017 & 19001 \\
20 & -0.73 & 19110 & 19095 \\
50 & -0.75 & 19061 & 19053 \\
100 & -0.76 & 19105 & 19096 \\
\hline
\end{tabular}

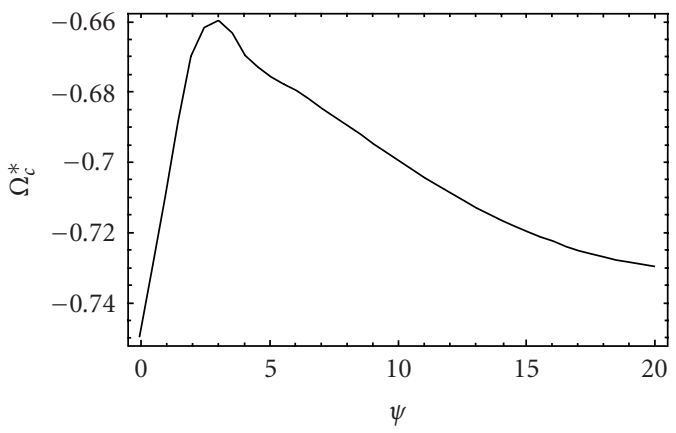

Figure 3.1. Variation of critical value $\Omega_{c}^{*}$ with magnetic field parameter $\psi$ at $\xi=0.95$ and $\varphi=0.2$.

value $\Omega_{c}^{*}$ to be approximately -0.75 and found that it is independent of the magnitude of applied magnetic field.

The ratio $T^{*}=T_{c \sigma} / T_{0}$, where $T_{c \sigma}$ is the critical Taylor number at a given value of $\sigma$ and $T_{0}=T_{c}$ at $\sigma=0$, has been found numerically at $\Omega^{*}=-1$ and $\psi=0$ and 6 , The points in $\left(\sigma, T^{*}\right)$ plane have been plotted in Figure 3.2. In the absence of magnetic field, the critical disturbance is nonaxisymmetric with critical azimuthal wave number $\sigma_{c}=4$. The critical disturbance remains nonaxisymmetric at $\psi=6$ but occurs at $\sigma_{c}=5$. Thus, the magnetic field affects the value of critical azimuthal wave number.

Paying attention to the variation of $T_{c}$ with magnetic field at $\Omega^{*}=-1$, we have found that the critical disturbance remains nonaxisymmetric for all values of $\psi$. For $2 \leq \psi \leq$ 6.8 approximately, the critical nonaxisymmetric mode of disturbance occurs at $\sigma_{c}=5$. The difference in the corresponding values of $T_{c}$ at $\sigma=4$ and $\sigma=5$ is small whenever 


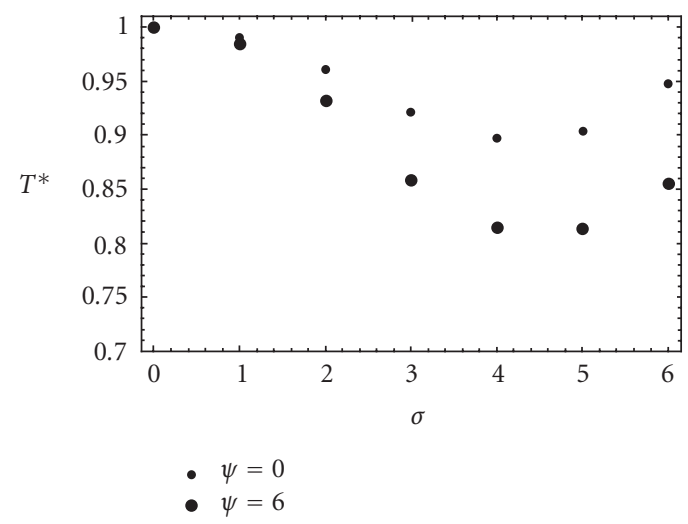

Figure 3.2. The plot of ratio $T^{*}=T_{c \sigma} / T_{0}$ at fixed values, $\xi=0.95, \varphi=0.2$, and $\Omega^{*}=-1$.

the value of magnetic field parameter is not too large. For $\left(\psi, \Omega^{*}\right)=(2,-1)$, the critical Taylor number $T_{c}$ for $\sigma=4$ has been found to be 26503, whereas it is 26403 at $\sigma=5$. For $\left(\psi, \Omega^{*}\right)=(6,-1), T_{c}$ is 33920 at $\sigma=4$ and it is 33871 at $\sigma=5$. On further increase of magnetic field parameter $\psi$, the critical nonaxisymmetric mode of disturbance occurs at $\sigma_{c}=4$.

Figure 3.3a shows variation of critical Taylor number $T_{c}$ with magnetic field parameter $\psi$. The two curves correspond to $\sigma=0$ and 4 , respectively. $T_{c}$ increases initially with $\psi$, attains a maximum, and then starts decreasing with further increase in $\psi$ until it becomes constant. Figure 3.3b shows the variation of $T_{c}$ with $\psi$ for $\sigma=0$ and 5 .

The corresponding variation of critical axial wave number $k_{c}$ and critical angular frequency $\omega_{c}$ with the magnetic field parameter $\psi$ at the onset of nonaxisymmetric mode of the Couette-Taylor instability have been shown in Figures 3.3c and 3.3d, respectively. Figure $3.3 \mathrm{c}$ shows that the critical axial wave number for nonaxisymmetric disturbance remains less than its corresponding values for axisymmetric disturbance for all values of the applied magnetic field. The plots show that $k_{c}$ at fixed $\sigma$ decreases initially with increase of the magnetic field parameter $\psi$, attains a minimum at certain value of $\psi$, starts increasing with further increase of $\psi$, and then becomes constant at high magnetic fields. A similar variation of the critical frequency parameter $\omega_{c}$ with magnetic field occurs, which has been observed from Figure 3.3d.

\section{Conclusion}

We have investigated the stability of Couette flow of a ferrofluid with nonaxisymmetric disturbance in the presence of axial magnetic field. We have taken into consideration the magnetic field perturbations in ferrofluid in the gap between cylinders. A general analysis has been done and throughout the investigation, no approximations for the narrow-gap limit have been assumed. The magnetic field causes the critical value of parameter $\Omega^{*}$ to vary. The critical value of the angular speed ratio $\Omega^{*}$ for change of the mode of instability from axisymmetry to nonaxisymmetry in the Couette flow is close to -0.75 at small and 


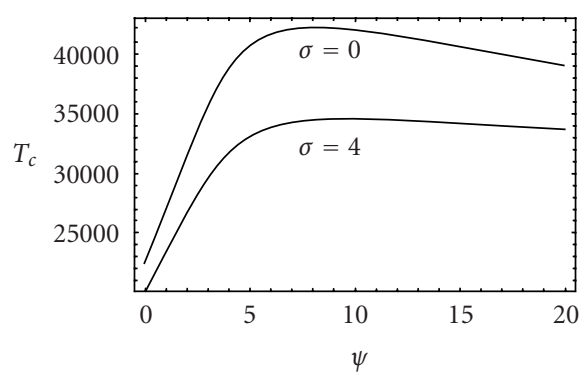

(a)

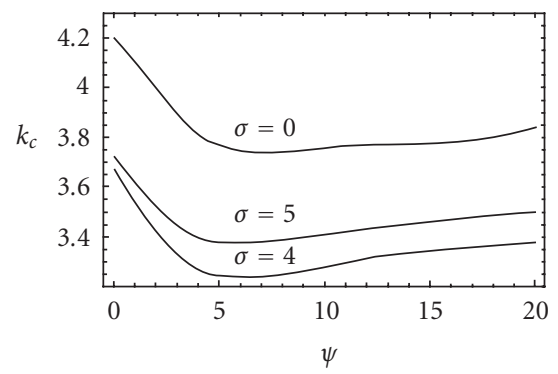

(c)

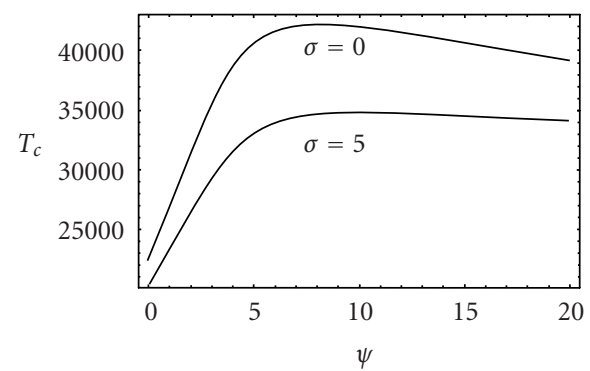

(b)

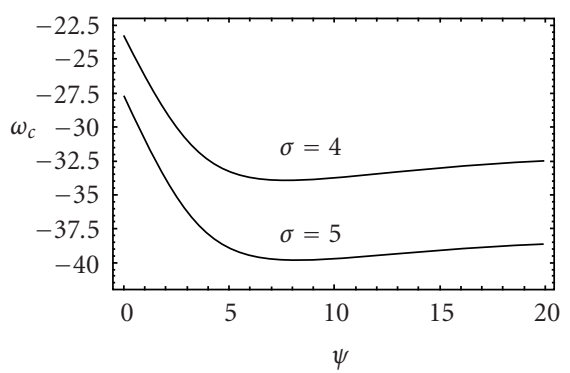

(d)

Figure 3.3. (a) Variation of the critical Taylor number $T_{c}$ with magnetic field parameter $\psi$ at fixed values: $\xi=0.95, \varphi=0.2$, and $\Omega^{*}=-1$ for two different values of azimuthal wave number $\sigma=0,4$. (b) Variation of the critical Taylor number $T_{c}$ with magnetic field parameter $\psi$ at fixed values: $\xi=0.95$, $\varphi=0.2$, and $\Omega^{*}=-1$ for two different values of azimuthal wave number $\sigma=0,5$. (c) Variation of critical axial wave number $k_{c}$ with $\psi$ at $\xi=0.95, \varphi=0.2$, and $\Omega^{*}=-1$ at different values of azimuthal wave number $\sigma=0,5,4$. (d) Variation of angular frequency of critical disturbance for the fixed values the same as for Figure 3.3c at $\sigma=4,5$.

high values of applied magnetic field but varies at intermediate magnetic fields. Applied magnetic field causes the change of critical azimuthal wave number $\sigma_{c}$. The onset of nonaxisymmetric mode of instability in the Couette flow of a ferrofluid occurs at $\sigma=5$ for intermediate values of applied magnetic field at $\Omega^{*}=-1$. For high values of applied magnetic field, when the ferrofluid magnetization approaches its saturation value the onset of nonaxisymmetric mode of instability occurs at $\sigma=4$.

\section{References}

[1] V. G. Bashtovoy, B. M. Berkowsky, and A. N. Vislovich, Introduction to Thermomechanics of Magnetic Fluids, Springer, Berlin, 1988.

[2] E. Blums, A. Cebers, and M. M. Maiorov, Magnetic Fluids, Walter de Gryuter, Berlin, 1997.

[3] S. Chandrasekhar, Hydrodynamic and Hydromagnetic Stability, The International Series of Monographs on Physics, Clarendon Press, Oxford, 1961. 
[4] M. H. Chang, C.-K. Chen, and H. C. Weng, Stability of ferrofluid flow between concentric rotating cylinders with an axial magnetic field, Internat. J. Engrg. Sci. 41 (2003), no. 2, 103-121.

[5] D. Coles, Transition in circular Couette flow, J. Fluid Mech. 21 (1965), no. 3, 385-425.

[6] R. C. Di Prima, Application of the Galerkin method to problems in hydrodynamic stability, Quart. Appl. Math. 13 (1955), 55-62.

[7] Stability of nonrotationally symmetric disturbances for viscous flow between rotating cylinders, Phys. Fluids 4 (1961), no. 6, 751-755.

[8] R. J. Donnelly, Experiments on the stability of viscous flow between rotating cylinders. I. Torque measurement, Proc. Roy. Soc. London Ser. A 246 (1958), no. 1246, 312-325.

[9] D. L. Harris and W. H. Reid, On the stability of viscous flow between rotating cylinders. II. Numerical analysis, J. Fluid Mech. 20 (1964), part 1, 95-101.

[10] E. R. Krueger, A. Gross, and R. C. Di Prima, On the relative importance of Taylor-Vortex and non-axisymmetric modes in flow between rotating cylinders, J. Fluid Mech. 24 (1966), no. 3, 521-538.

[11] M. Niklas, H. Müller-Krumbhaar, and M. H. Lücke, Taylor-vortex flow of ferrofluids in the presence of general magnetic fields, J. Magn. Magn. Mat. 81 (1989), no. 1-2, 29-38.

[12] R. E. Rosenswieg, Ferrohydrodynamics, Cambridge University Press, Cambridge, 1985.

[13] M. I. Shliomis, Effective viscosity of magnetic suspensions, Sov. Phys. JETP 34 (1972), 1291-1294.

[14] J. Singh and R. Bajaj, Couette flow in ferrofluids with magnetic field, J. Magn. Magn. Mat. 294 (2005), no. 1, 53-62.

[15] G. I. Taylor, Stability of a viscous liquid contained between two rotating cylinders, Philos. Trans. Roy. Soc. London Ser. A 223 (1923), 289-343.

Jitender Singh: Centre for Advanced Study in Mathematics, Panjab University, Chandigarh 160014, India

E-mail address: sonu_maths@yahoo.com

Renu Bajaj: Centre for Advanced Study in Mathematics, Panjab University, Chandigarh 160014, India

E-mail address: renubajaj20012000@yahoo.com 


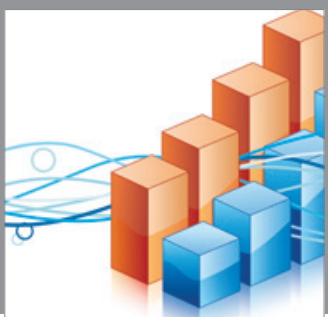

Advances in

Operations Research

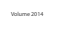

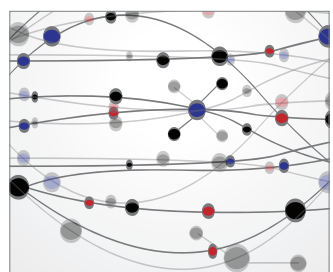

\section{The Scientific} World Journal
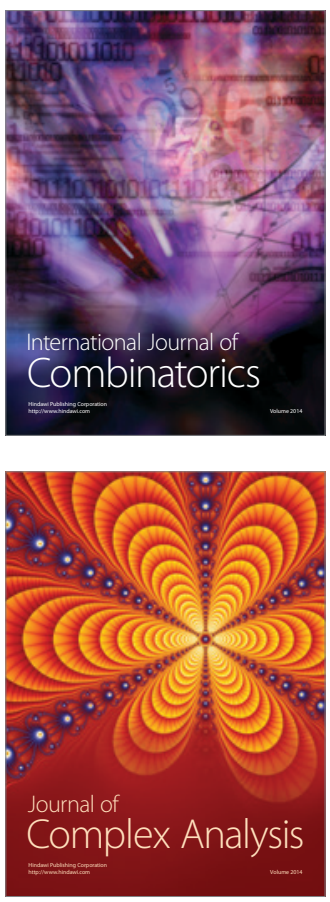

International Journal of

Mathematics and

Mathematical

Sciences
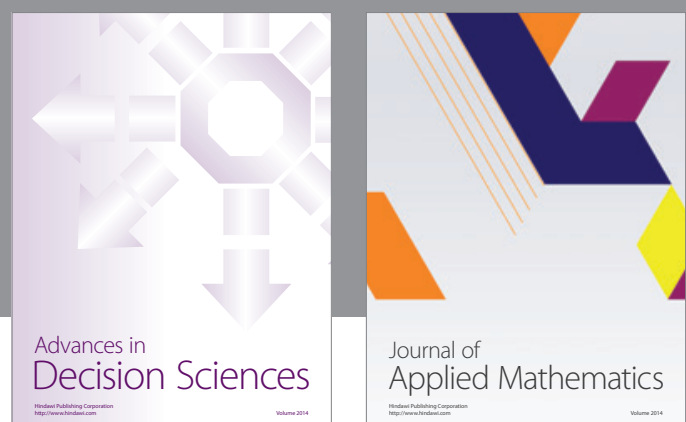

Journal of

Applied Mathematics
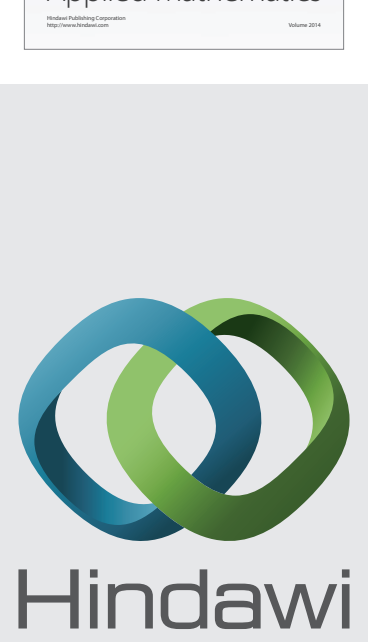

Submit your manuscripts at http://www.hindawi.com
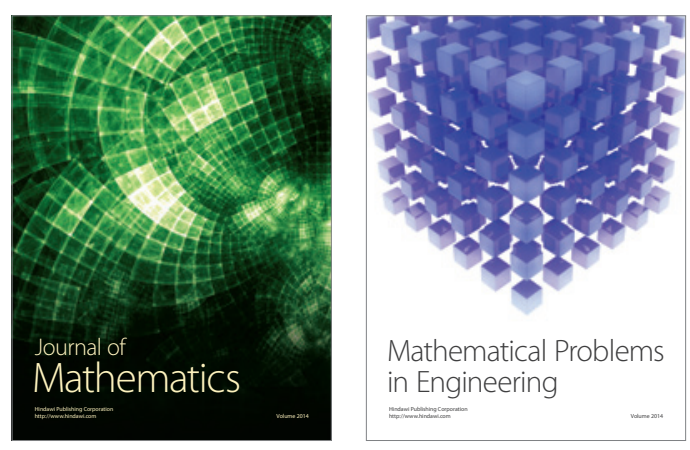

Mathematical Problems in Engineering
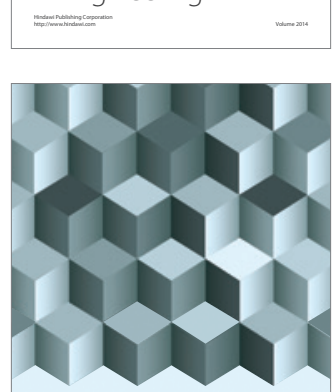

Journal of

Function Spaces
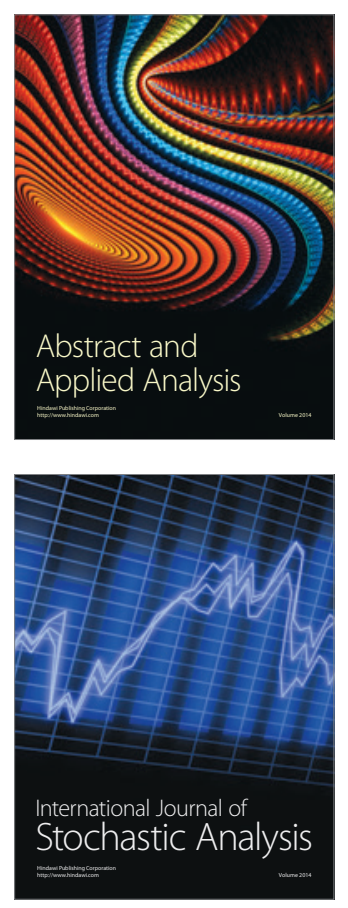

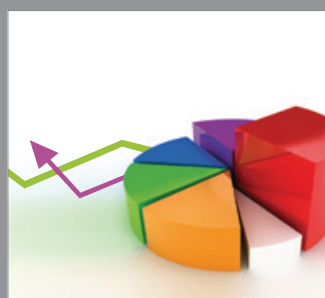

ournal of

Probability and Statistics

Promensencen
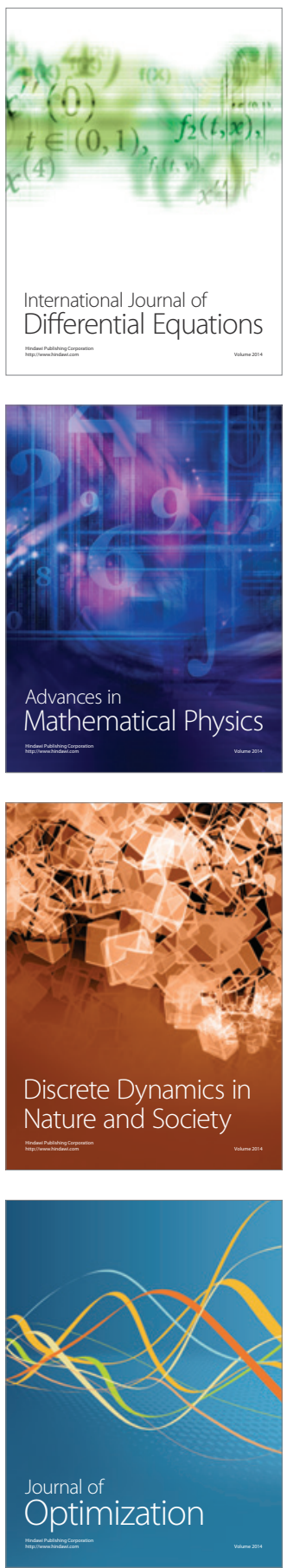